\title{
Implantatinsertion direkt durch die Schablone
}

Mit Einführung der neuen Implantatverpackung für alle BEGO Semados ${ }^{\circledR}$ Implantate wurde auch der Implantateinbringpfosten auf die Vollnavigation angepasst. Der Durchmesser des neuen Pfostens entspricht exakt der Geometrie der BEGO Guide Masterhülsen, sodass eine Insertion des Implantats durch die Schablone möglich ist. Mit den BEGO Guide Trays stehen die notwendigen Instrumente hierzu zur Verfügung. Der BEGO Guide Connector ist das neue universelle Einbringwerkzeug für die Implantateinbringung durch eine Schablone. In Abhängigkeit von der Implantatlänge wird ein Tiefenstopp eingestellt, um das Implantat exakt auf die vorab geplante Tiefe einbringen zu können. Eine Spezialität der Trays sind zudem die zum Patent ange- meldeten Masterhülsen mit integriertem Arretierungsmechanismus für die BEGO Guide Spoons. Diese Reduktionshülsen lassen sich in der Schablone fixieren, sodass sie beim Bohrvorgang nicht fixiert werden müssen. Dies sorgt für ein komfortables Handling.Beide Chirurgietrays sind mit allen gängigen Planungs- und Schablonensystemen kompatibel und eine zusätzliche Software wird nicht benötigt. Anwender ohne eigene Planungssoftware können zudem eine Implantatplanung mit Bohrschablone einfach und preiswert über das BEGO Guide Planungszentrum (guide.bego.com) beziehen. Interessierten Anwendern stellt die BEGO Implant Systems für die 1. Probe-OP ein Tray kostenfrei zur Verfügung. Weitere Informationen und Anmeldung zum Portal unter guide.bego.com, sowie auch telefonisch unter 0421 / 2028488.

Nach einer Pressemitteilung der BEGO Implant Systems GmbH \& Co. KG, Bremen

www.bego-implantology.com 